\title{
RELAÇÃO DOS ELEMENTOS BIOQUÍMICOS E FARMACOLÓGICOS COM O DESENVOLVIMENTO DE COMPLICAÇÕES CARDÍACAS EM PORTADORES DE COVID-19: REVISÃO DE LITERATURA
}

\author{
Relationship of biochemical and pharmacological elements with the development of cardiac \\ complications in patients with COVID-19: Literature Review
}

\author{
Bruna Katerine Goginho Gomes ${ }^{1}$ \\ Hilária Augusto Lopes Vieira ${ }^{2}$ \\ Ana Paula Ferreira Maciel ${ }^{3}$ \\ Vinícius Gomes e Martins ${ }^{4}$ \\ Aurelina Gomes e Martins ${ }^{5}$ \\ Fernanda Sabrina Dias dos Santos ${ }^{6}$ \\ Otávio Henrique Oliveira Macedo ${ }^{7}$ \\ Carla Silvana de Oliveira e Silva ${ }^{8}$
}

Resumo: Objetivo: Descrever os elementos bioquímicos e farmacológicos e sua relação com o desenvolvimento ou agravamento de alterações cardíacas em portadores de COVID-19. Método: Trata-se de uma revisão integrativa de literatura com busca de artigos em três bases de dados: Medical Literature Analysis and Retrieval System Online (MEDLINE), Literatura Latino-Americana em

\footnotetext{
${ }^{1}$ Enfermeira pela Universidade Estadual de Montes Claros (UNIMONTES). Montes Claros/MG- Brasil. $\square$ brunnahk@gmail.com. (1) https://orcid.org/0000-0002-5288-0332.

${ }^{2}$ Enfermeira pela Universidade Estadual de Montes Claros (UNIMONTES). Montes Claros/MG- Brasil. $\square$ hilariaaugusto.moc@gmail.com. (1) https://orcid.org/0000-0003-0399-594X.

${ }^{3}$ Mestre em Enfermagem pela Universidade Estadual de Montes Claros (UNIMONTES). Montes Claros/MGBrasil. $₫$ ana.maciel@unimontes.br. (1) https://orcid.org/0000-0001-8056-4022.

${ }^{4}$ Graduando de Medicina pelo Centro Universitário FIPMoc (UNIFIPMoc). Montes Claros MG- Brasil. viniciusgomesemartins@ @otmail.com. (I) https://orcid.org/0000-0001-8673-3659.

${ }^{5}$ Mestre em Enfermagem pela Universidade Federal de São Paulo (UNIFESP). São Paulo/SP - Brasil. aurelina2007@yahoo.com.br. (I) https://orcid.org/0000-0003-3582-8994.

${ }^{6}$ Graduanda do curso de Biomedicina da Faculdade de Saúde e Humanidades (FASI), Montes Claros MGBrasil. $\bowtie$ fernandasabrina.sabrina@gmail.com. (D) https://orcid.org/0000-0001-7852-7425.

${ }^{7}$ Enfermeiro pela Universidade Estadual de Montes Claros (UNIMONTES), Montes Claros MG- Brasil. $\square$ rickenfermagem@yahoo.com.br. 1 https://orcid.org/0000-0002-7502-5136.

8 Pós-doutorado pela Universidade Federal de Saõ Paulo (UNIFESP), São Paulo SP- Brasil. $\square$ carla.silva@unimontes.br. (D) https://orcid.org/0000-0002-2752-1557.

$\begin{array}{ccc}\text { Recebido em } & \text { Aceito em } & \text { Publicado em } \\ 04 / 01 / 2021 & 14 / 01 / 2021 & 30 / 05 / 2021\end{array}$
}


Ciências da Saúde (LILACS) e Coleciona SUS consultados através da Biblioteca Virtual em Saúde (BVS Brasil), no período de 01 a 18 de junho de 2020. Foi construído um fluxograma com as etapas de identificação, seleção e inclusão dos textos além de um quadro sinóptico que permitiu a visualização da estrutura e organização do conteúdo. Resultados: Portadores de doenças cardiovasculares, hipertensos e diabéticos apresentam maior expressão da ECA 2 o que contribuiu para elevar a chance de se infectarem pelo vírus. Não foi possível confirmar se os anti-hipertensivos estão relacionados com o desenvolvimento da COVID-19. Considerações finais: Existe relação entre a COVID-19 e o desenvolvimento e/ou agravamento de alterações cardiovasculares, esta relação pode ser intermediada pela ação da enzima conversora da angiotensina (ECA 2) e níveis elevados dos dímeros D.

Palavras-Chave: COVID-19; SARS-CoV-2; Doenças Cardiovasculares.

Abstract: Justification and Objectives: The choice for this theme is important because it is a recently discovered disease that is generating a great impact worldwide, and is therefore of high scientific relevance. The objective was to describe the biochemical and pharmacological elements and their relationship with the development or worsening of cardiac changes in patients with COVID-19. Content: This is an integrative literature review with search for articles in three databases: Medical Literature Analysis and Retrieval System Online (MEDLINE), Latin American Literature in Health Sciences (LILACS) and SUS collections consulted through the Library Virtual Health (BVS Brasil), from June 1 to $18,2020.11$ articles comprised this review. A flowchart was made showing the following topics: data collection; selection of records; analysis of the selection by title or abstract; eligibility assessment; studies included for the construction of the synoptic framework. Conclusion: There is a relationship between COVID-19 and the development and / or worsening of cardiovascular changes, this relationship can be mediated by the action of the angiotensin-converting enzyme (ACE 2) and elevated levels of D-dimers. Patients with cardiovascular, hypertensive and diabetics have higher expression of ACE 2, which contributed to increase the chance of becoming infected by the virus. It was not possible to confirm whether antihypertensive drugs are related to the development of COVID-19.

Descriptors: COVID-19; SARS-CoV-2; Cardiovascular diseases 


\section{INTRODUÇÃO}

Nas últimas duas décadas, em eventos distintos, surgiram dois vírus altamente morbíparos que são o Coronavírus causador da Síndrome Respiratória Aguda Grave (SARSCoV-2) e o Coronavírus da Síndrome Respiratória do Oriente Médio (MERS-CoV). Ambos causam infecção do trato respiratório inferior como também manifestações extrapulmonares que podem ser fatais. ${ }^{1,2,3}$

Em dezembro de 2019, um grupo pessoas de Wuhan na China foi diagnosticada com uma espécie de pneumonia aguda, de causa desconhecida. ${ }^{4,5}$ Depois da realização de inúmeros exames uma nova cepa de Coronavírus foi confirmada em três indivíduos. ${ }^{6-9}$

Em 9 de janeiro de 2020, a Organização Mundial da Saúde (OMS) confirmou a circulação deste vírus ${ }^{10,11}$ classificado de Betacoronavírus e denominado provisoriamente de Novel Coronavirus Infected Pneumonia (2019nCoV). Em 30 de janeiro de 2020, a OMS anunciou que se tratava de uma epidemia e em 11 de fevereiro o vírus foi oficialmente nomeado Síndrome Respiratória Aguda Grave causada pelo SARS-CoV-2 ou COVID-19.,2,12

Esta doença se espalhou rapidamente ${ }^{13}$ pelo mundo, sendo identificado em outros países, ${ }^{14}$ inclusive no Brasil. Desta forma, em 11 de março ela passou a ser considerada uma pandemia pela Organização Mundial de Saúde. ${ }^{15,14,7,10}$

Observou-se que os principais sintomas apresentados por portadores do COVID19 são respiratórios, mas, que alguns pacientes apresentavam graves danos cardiovasculares $^{13,16}$. Além disso, que pacientes com doenças cardiovasculares (DC) preexistentes apresentavam descompensação cardíaca acentuada o que aumentava o risco de morte. ${ }^{17-18,19}$ Pesquisadores revisaram a literatura clínica e biomédica disponível sobre os riscos cardiovasculares da COVID-19 e compreenderam que naquele momento ainda permanecia desconhecido como o SARS-CoV-2 estava contribuindo para a doença cardíaca. $^{20,21}$

Diante do exposto, levantou-se o seguinte problema de pesquisa: "Quais fatores bioquímicos e farmacológicos estão relacionados com complicações cardíacas presentes em portadores de COVID-19?". Objetivou-se então descrever a relação entres os elementos bioquímicos e farmacológicos com o desenvolvimento de complicações cardíacas em portadores de COVID-19. 
A escolha por este tema se faz importante por esta ser uma doença recentemente descoberta que está gerando um grande impacto a nível mundial, sendo desta forma, de alta relevância científica.

\section{MÉTODO}

Trata-se de uma revisão integrativa de literatura pautada na: 1) elaboração de uma questão de pesquisa orientadora da estratégia de busca; 2) variedade de fontes para a localização dos estudos; 3) definição de critérios de inclusão e exclusão; e 4) avaliação da qualidade metodológica das produções recuperadas.

O levantamento dos artigos foi realizado em três bases de dados: Medical Literature Analysis and Retrieval System Online (MEDLINE), Literatura Latino-Americana em Ciências da Saúde (LILACS) e Coleciona SUS consultados através da Biblioteca Virtual em Saúde (BVS Brasil), no período de 01 a 18 de junho de 2020.

Para seleção dos estudos foram utilizados os descritores COVID-19, SARS-CoV2 e doenças cardiovasculares presentes nos Descritores em Ciências da Saúde (DeCS). Estes foram pareados utilizando-se o operador boleano "AND" o qual define relações entre termos em uma pesquisa.

Foram incluídos trabalhos publicados em qualquer local do mundo, entre os anos de 2019 e 2020, nos idiomas português ou inglês e que estivessem disponíveis na íntegra.

Como critérios de exclusão foram desconsiderados capítulos de livros, editoriais, entre outros formatos de textos, por não passarem por processo rigoroso de avaliação por pares, como ocorre com os artigos científicos. Excluíram-se ainda teses, dissertações, monografias, estudos incompreensíveis quando traduzidos e artigos repetidos em mais de uma base de dados, neste caso, foi considerada somente quando apareceu pela primeira vez para análise.

O levantamento dos dados bibliográficos foi realizado por dois autores/pesquisadores, com base nos critérios de inclusão estabelecidos. A primeira etapa de seleção das produções foi realizada mediante a leitura e a análise dos títulos e resumos de todos os artigos identificados. Após essa triagem inicial, na segunda etapa, procedeu-se à leitura na íntegra dos estudos selecionados, a qual possibilitou que outros textos também 
fossem excluídos por não atenderem à proposta da revisão. Na terceira etapa, as principais informações dos artigos foram sintetizadas em uma planilha para que pudessem orientar as análises descritivas e críticas dos estudos selecionados.

Tendo em vista que este trabalho é uma revisão integrativa ele está isento de revisão ética.

\section{RESULTADO}

O levantamento bibliográfico localizou 70 estudos, que após passarem pela filtragem dos critérios de inclusão e exclusão resultou em 11 artigos, a maioria dos estudos era descritivo, do tipo revisão de literatura, publicados principalmente em 2020. Todos os estudos encontravam-se disponíveis em revistas indexadas na base de dados MEDLINE. Foi construído um fluxograma com as etapas de identificação, seleção e inclusão dos textos além de um quadro sinóptico que permitiu a visualização da estrutura e organização do conteúdo.

O levantamento bibliográfico localizou 70 resultados, dos quais 14 foram excluídos após leitura do título e dos resumos, 33 foram descartados após filtragem pelos critérios de inclusão estabelecidos e 12 foram excluídos após leitura na íntegra (seis por apresentarem informações repetidas, dois estudos que ficaram confusos após a tradução e quatro que não correspondiam adequadamente ao tema. Os 11 artigos restantes compuseram o corpus de análise da revisão, sendo publicados principalmente no ano de 2020.

Todos os estudos encontravam-se disponíveis em revistas indexadas na base de dados MEDLINE, o que deixa nítido sua excelência em publicações internacionais na área da saúde. Estes foram baixados no formato PDF e traduzidos do inglês para o português pelo DocTranslato, um programa online e gratuito.

A Figura 1 apresenta o fluxograma com as etapas de identificação, seleção e inclusão dos textos, com os seguintes tópicos: coleta de dados; seleção dos registros; análise da seleção por título ou resumo; avaliação de elegibilidade; estudos incluídos para construção do quadro sinóptico.

Quanto ao delineamento, a maioria dos estudos era descritivo, do tipo revisão de literatura (Figura 1). 
FIGURA 1 - Diagrama de análise de processo de seleção dos artigos para revisão integrativa, Montes Claros- MG 2020.

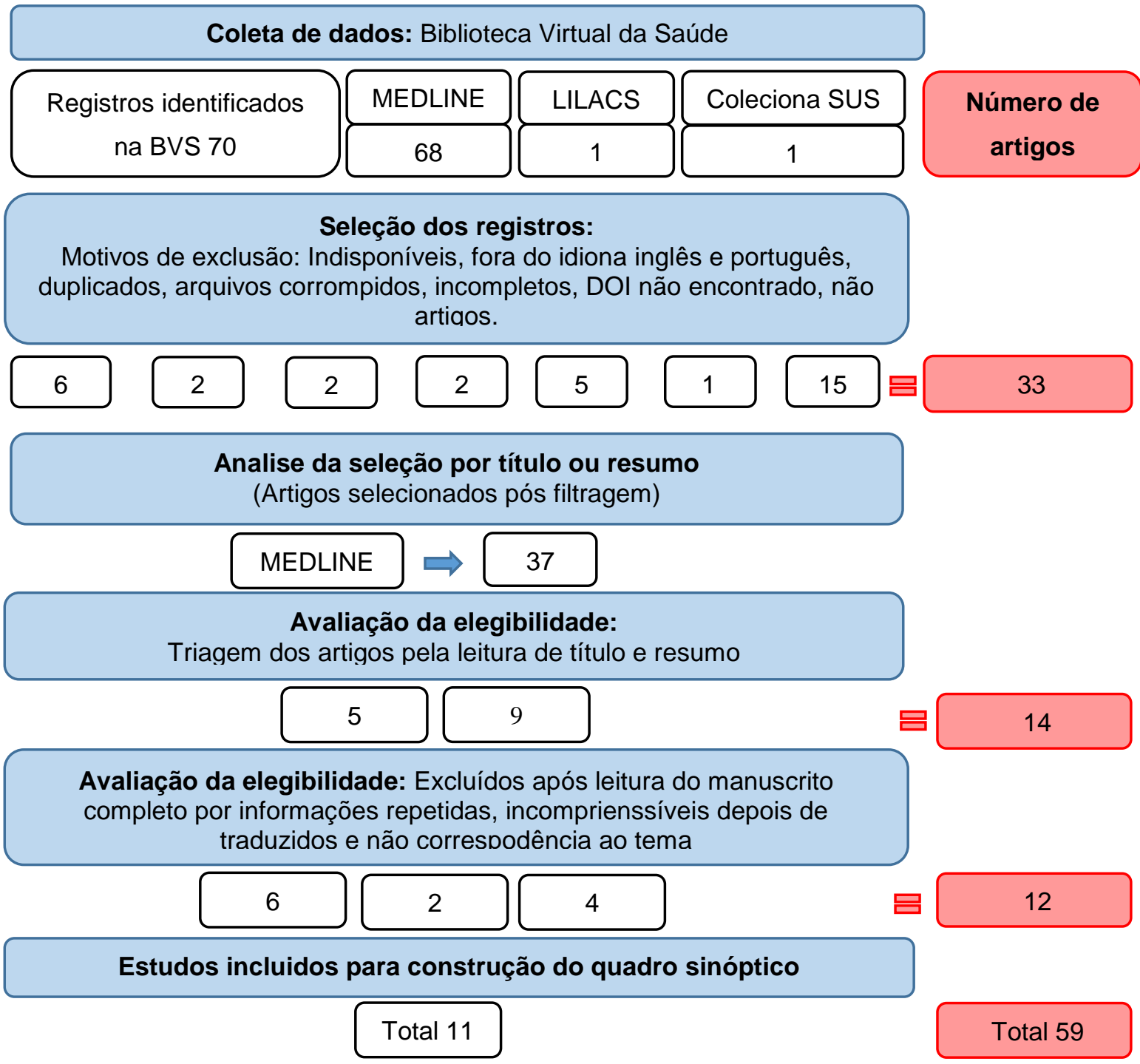

Fonte: GOMES, BKG et al., 2020. 2020.

Posteriormente, durante a leitura dos artigos escolhidos, foi construído um quadro sinóptico (quadro 1) que permitiu a visualização da estrutura e organização do conteúdo, o mesmo apresenta as seguintes informações: autores, título do artigo, base indexadora, ano de publicação, revista, procedência, objetivo do estudo e metodologia utilizada. 


\begin{tabular}{|c|c|c|c|c|c|c|c|}
\hline I & $\begin{array}{l}\text { Akhmerov } \\
\text { A e } \\
\text { Marbán E }\end{array}$ & COVID-19 and the Heart & $\begin{array}{l}\text { MEDLIN } \\
\text { E, } 2020\end{array}$ & $\begin{array}{l}\text { Circ Res } \\
\text { A1 }\end{array}$ & $*$ & $\begin{array}{l}\text { Revisar o que se sabe sobre a } \\
\text { fisiopatologia do COVID-19, suas } \\
\text { manifestações cardiovasculares e } \\
\text { perspectivas terapêuticas emergentes. }\end{array}$ & $\begin{array}{ll}\text { Revisão } & \text { de } \\
\text { literatura } & \end{array}$ \\
\hline II & $\begin{array}{l}\text { Gheblawi } \\
\text { M, et al. }\end{array}$ & $\begin{array}{l}\text { Angiotensin-Converting Enzyme } \\
\text { 2: SARS-CoV-2 } \\
\text { Receptor and Regulator of the } \\
\text { Renin-Angiotensin } \\
\text { System }\end{array}$ & $\begin{array}{l}\text { MEDLIN } \\
\text { E, } 2020\end{array}$ & $\begin{array}{l}\text { Circ Res } \\
\text { A1 }\end{array}$ & $*$ & $\begin{array}{l}\text { Revisar o papel crítico da ACE2 } \\
\text { Como o novo receptor SARS-CoV-2 } \\
\text { e como regulador negativo do } \\
\text { sistema junina-angiotensina, } \\
\text { juntamente com implicações para a } \\
\text { pandemia da doença de coronavírus } \\
2019 \text { e doenças cardiovasculares } \\
\text { associadas. }\end{array}$ & $\begin{array}{ll}\text { Revisão } & \text { de } \\
\text { literatura } & \end{array}$ \\
\hline IV & $\begin{array}{l}\text { Iaccarino } \\
\mathrm{G}, \text { et al. }\end{array}$ & $\begin{array}{l}\text { Renin-Angiotensin System } \\
\text { Inhibition in Cardiovascular } \\
\text { Patients at the Time of COVID19: } \\
\text { Much Ado for Nothing? A } \\
\text { Statement of Activity from the } \\
\text { Directors of the Board and the } \\
\text { Scientific Directors of the Italian } \\
\text { Society of Hypertension. }\end{array}$ & $\begin{array}{l}\text { MEDLIN } \\
\text { E, } 2020\end{array}$ & $\begin{array}{l}\text { High } \\
\text { Blood } \\
\text { Press } \\
\text { Cardiova } \\
\text { sc Prev } \\
\text { B2 }\end{array}$ & Itália & $\begin{array}{l}\text { Apresentar declaração fundamentada } \\
\text { da Sociedade Italiana de Hipertensão } \\
\text { para manter os tratamentos anti- } \\
\text { hipertensivos em andamento }\end{array}$ & $\begin{array}{l}\text { Pesquisa } \\
\text { Documental }\end{array}$ \\
\hline
\end{tabular}




\section{Continua...}

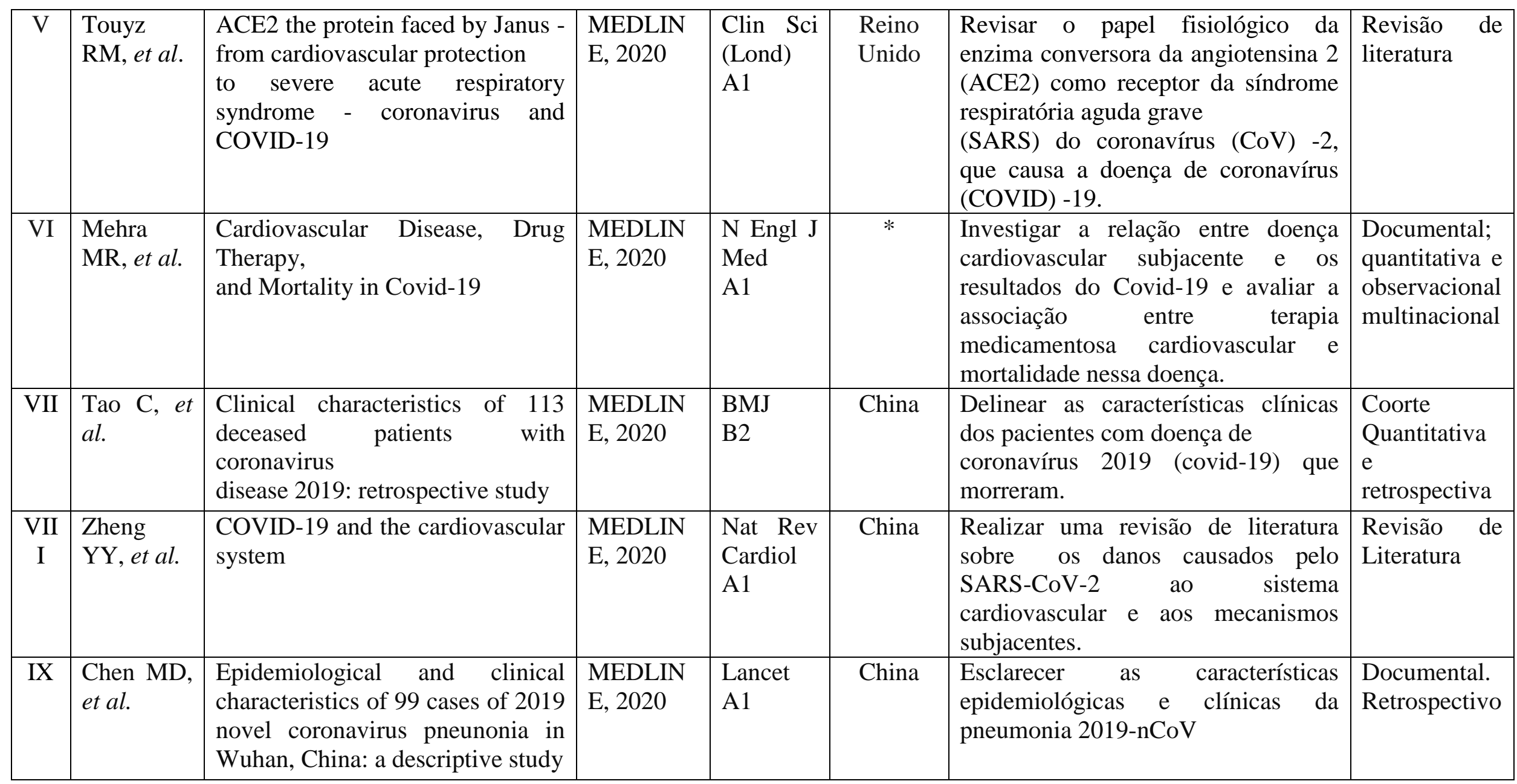

Continua... 


\begin{tabular}{|c|c|c|c|c|c|c|c|}
\hline$X$ & $\begin{array}{l}\text { Cheng } \mathrm{P}, \\
\text { et al. }\end{array}$ & $\begin{array}{l}\text { Cardiovascular Risks in Patients } \\
\text { with COVID-19: Potential } \\
\text { Mechanisms and Areas of } \\
\text { Uncertainty }\end{array}$ & $\begin{array}{l}\text { MEDLIN } \\
\text { E, } 2020\end{array}$ & $\begin{array}{l}\frac{\text { Curr }}{\text { Cardiol }} \\
\frac{\text { Rep }}{\text { B2 }}\end{array}$ & $\begin{array}{l}\text { Estados } \\
\text { Unidos }\end{array}$ & $\begin{array}{l}\text { Revisar a literatura clínica e } \\
\text { biomédica disponível sobre os riscos } \\
\text { cardiovasculares do COVID-19 }\end{array}$ & $\begin{array}{ll}\text { Revisão } & \text { de } \\
\text { literatura } & \end{array}$ \\
\hline
\end{tabular}

Legenda: * País de afiliação não apresentado na base de dados.

Fonte: GOMES BKG, et al., 2020. 


\section{DISCUSSÂO}

As investigações selecionadas para esta revisão integrativa de literatura demonstraram uma boa qualidade metodológica, o que garante maior confiabilidade nos resultados obtidos.

O estudo II, mostrou envolvimento cardíaco na COVID-19 em 7 de 20 amostras de autópsia de tecido cardíaco de pacientes que vieram a óbito. Nestas autópsias foram percebidas fibrose cardíaca e inflamação e derrames pleurais bilaterais. ${ }^{22}$

Pesquisa quantitativa e retrospectiva realizada na China, a qual delineou as características clínicas dos pacientes com COVID-19 evidenciou que entre os 113 pacientes incluídos no estudo e que vieram a óbito as comorbidades mais comuns foram os problemas cardíacos (lesão cardíaca aguda (72/94; 77\%), insuficiência cardíaca (41/83; 49\%). As complicações cardíacas também foram frequentes nos pacientes sem comorbidades, sugerindo que o alto risco de complicações cardíacas em pacientes falecidos não poderia ser inteiramente atribuído à doença cardiovascular pré-existente. ${ }^{1}$

$\mathrm{O}$ artigo XI mostrou que o dímero-D em níveis elevados estava associado à mortalidade, em até 28 dias, para pacientes com COVID-19 que apresentaram esta infecção ou quadro séptico ainda no pronto-socorro. Os mecanismos relacionados foram as respostas sistêmicas às citocinas pró-inflamatórias mediadas pela placa de aterosclerose, o que contribuiu diretamente para sua ruptura gerada pela inflamação local, indução de fatores prócoagulantes e alterações hemodinâmicas, que predispõem à isquemia e trombose. ${ }^{23}$

Estudo desenvolvido na China observou que mais da metade dos pacientes (99) com COVD-19 acompanhados do dia 01 a 25 de janeiro eram portadores de alguma doença crônica, entre elas cardiopatias. Os exames confirmaram Síndrome Coronariana Aguda (SCA) devido à elevação dos dímeros-D que refletem o risco aumentado de trombose. ${ }^{2}$

Este achado é confirmado no artigo I, a SCA e o Infarto Agudo do Miocárdio (IAM) podem surgir em pacientes com COVID-19, porém, a incidência de tais eventos ainda não estão bem determinados. Em princípio, o risco de SCA em pacientes infectados pode aumentar devido à maior tendência à trombose evidenciado por níveis significativamente elevados de dímero -D. A possibilidade é que os mecanismos da lesão cardíaca envolvem, dano indireto da resposta inflamatória sistêmica, aumento do estresse cardíaco devido a 
insuficiência respiratória e hipoxemia, infecção miocárdica direta por SARS-CoV-2, ou uma combinação de todas essas causas. ${ }^{24}$

Em um artigo multinacional que investiga a relação entre doença cardiovascular subjacente e os resultados do Covid-19 afirma que a disfunção das células endoteliais vasculares, depressão miocárdica associada à inflamação, cardiomiopatia por estresse, infecção viral direta no coração e vasos ou a resposta do hospedeiro podem causar ou piorar a insuficiência cardíaca. ${ }^{12}$

Pacientes portadores de SCA infectados com SARS-CoV-2 geralmente apresentam um prognóstico ruim. A reserva funcional cardíaca pode ser reduzida devido a isquemia ou necrose do miocárdio sendo mais provável que a insuficiência cardíaca ocorra, levando a uma deterioração repentina na condição desses pacientes levando a óbito. ${ }^{25}$

Pesquisas apontaram que a enzima conversora da angiotensina (ECA 2) (ACE 2) age como um receptor para o SARS-CoV-2 ${ }^{26}$. Sabe-se que o papel desta enzima no organismo humano está claramente descrito na literatura internacional, e segundo o artigo I esta é uma carboxipeptidase que tem a função de converter a angiotensina (Ang) II em Ang (1-7). Esta enzima é homóloga à ECA, porém desempenha um papel de contrapeso no sistema renina-angiotensina-aldosterona que é um poderoso mecanismo de controle da pressão arterial pela homeostase cardiovascular. ${ }^{24}$

Ficou recentemente demonstrado que a ECA2 funciona como um receptor funcional para o Coronavírus da Síndrome Respiratória Aguda Grave (SARS-CoV2), agente responsável pela doença COVID-19. Observou-se ainda que, apesar da ECA 2 facilitar a entrada do vírus na superfície epitelial celular o que leva ao agravamento da insuficiência cardíaca (IC), hipertensão sistêmica e pulmonar (HP), infarto agudo do miocárdio (IAM) e diabetes, ela também tem a capacidade de oferecer proteção contra a lesão pulmonar aguda. ${ }^{22}$

Desta forma, doentes cardiovasculares, hipertensos e diabéticos, apresentam uma maior chance de serem infectados pelo COVID-19 em função da ECA 2 apresentar-se de forma mais expressa nesses pacientes, sendo encontrada em vários tecidos, mas predominantemente no fígado, intestino, rins, pulmões e coração. ${ }^{27,24,12}$

Os estudos relataram também que a utilização de duas classes de medicamentos anti-hipertensivos, inibidores da enzima conversora de angiotensina (iECA) e bloqueadores dos receptores de angiotensina (BRAs), levam ao aumento da expressão de ECA $2^{28}$ através 
de um mecanismo compensatório e com isto permite a entrada do SARS-CoV-2 na célula. Este aumento da expressão é feito principalmente por meio da ligação da proteína viral com o receptor ECA2, o que favorece tanto o desenvolvimento da infecção quanto a gravidade desta doença. $^{27}$

$\mathrm{O}$ estudo $\mathrm{V}$, também demostrou relação entre os iECA e os BRAs podem aumentar o risco de infecção por COVID-19 por meio da regulação positiva da ECA2. Entretanto, estes autores relatam que os BRAs, podem ter benefício terapêutico na COVID19 em função da resposta do hospedeiro ao vírus. Os BRAs conhecidamente elevam a expressão e atividade da ECA2 e este, por sua vez, geram um aumento na produção de Ang (1-7), um protetor tecidual, inibem ainda, a inflamação induzida por Ang 2 e a lesão aguda nos pulmões. ${ }^{29}$

Estudo multinacional, realizado em 169 hospitais na Ásia, Europa e América do Norte com 8.910 pacientes, que resultaram em óbito ou cura, coloca que o uso de iECA associou-se a uma melhor chance de sobrevida entre pacientes com Covid-19, mas, essa associação não foi encontrada com uso dos BRAs. No entanto, deve ser considerada com extrema cautela em função deste não ser do tipo clínico, com randomização e controle. ${ }^{12}$

Em contrapartida, estudo documental realizado na Itália, relata que para a Sociedade Italiana de Hipertensão (SIIA), não existe evidências clínicas em humanos para associar a hipertensão e outras doenças cardiovasculares (DCV) à COVID-19 e que não é possível afirmar que os iECA ou BRAs podem melhorar ou piorar a suscetibilidade para a doença. Coloca ainda que, o tratamento de cardiopatas com estas medicações deve ser mantido pois estes medicamentos são fundamentais para o controle das DCV e de difícil substituição. ${ }^{30}$

Dois grupos de medicamentos foram descritos como indicação para o tratamento da COVI-19, antivirais e antimaláricos, mas, autores apontam que este tratamento não está isento de toxicidade cardíaca e que esta pode ser diagnosticada através da presença de anormalidades de condução e síndrome do QT longo (Distúrbio do ritmo cardíaco que pode causar taquicardia grave). Colocam ainda que, com este tratamento, a resposta inflamatória do hospedeiro pode amplificar, mesmo com a diminuição da carga viral, e resultar em uma inflamação sistêmica que se inicia e alastra no órgão alvo (pulmão) com efeitos patológicos também sobre outros órgãos (coração) devido a alta resposta inflamatória gerada. ${ }^{24}$ 
Os pacientes em uso de antivirais devem ser monitorizados continuamente para controle dos riscos. ${ }^{25}$

\section{CONSIDERAÇÕES FINAIS}

Observou-se que existem algumas variações nos resultados apresentados à partir da revisão de literatura realizada. Importante destacar que existe relação entre a COVID-19 e o desenvolvimento e/ou agravamento de complicações cardiovasculares e que esta relação pode ser intermediada pela ação da ECA 2, níveis elevados dos dímeros D.

Portadores de doenças cardiovasculares, hipertensos e diabéticos apresentam maior expressão desta enzima (ECA 2) o que contribuiu para elevar a chance de se infectarem pelo vírus. Destaca-se que este fato não é genérico, uma vez que os doentes cardíacos são mais vulneráveis para qualquer outra doença, afinal o coração encontra-se fragilizado.

Portadores de cardiopatias infectados pelo SARS-CoV-2 apresentam risco de morte mais alto, este fato parece estar relacionado à maior tendência à trombose evidenciada por níveis elevados de dímero -D.

Através deste estudo não foi possível confirmar se os medicamentos iECA e BRA estão relacionados com o desenvolvimento da COVID-19, levantou-se apenas a hipótese destes medicamentos contribuem para o agravamento de seus sintomas.

Portanto, destaca-se a necessidade da realização de novos estudos com alto rigor metodológico para aprofundar os mecanismos potenciais que relacionam a COVID-19 com as complicações cardiovasculares.

\section{REFERÊNCIAS}

1. TAO, C, et al. Clinical characteristics of 113 deceased patients with coronavirus disease 2019: Retrospective study. BMJag., China. 12(1):3-17, mar.,2020. doi:https://doi.org/10.1136/bmj.m1091. Access in: 16 jun. 2020.

2. CHEN, et al., Epidemiological and clinical characteristics of 99 cases of 2019 novel coronavirus pneumonia in Wuhan, China: a descriptive study. Mag. Lancet Published Online 395: 507-13., January, 2020. doi:https://doi.org/10.1016/S01406736(20)30211-7 . Access en: 16 jun.2020.

3. ZAID, A, et al. Letter to the Editor: Angiotensin-converting enzyme 2: an ally or a Trojan horse? Implications in cardiovascular complications related to SARS-CoV-2. 
American Physiological Society, Jerusalém, Israel. 318(5):1080-83, mar., 2020. doi:https://doi.org/10.1152/ajpheart.00215.2020. Access in: 16 ago. 2020.

4. HUANG, Chaolin et al., Clinical features of patients infected with 2019 novel coronavirus in Wuhan, China. Lancet Published Online 395: 497-506., Jan., 2020. doi:https://doi.org/10.1016/S0140-6736(20)30183-5. Access in: 16 ago.2020.

5. DAVID, S. Hui et al., The continuing 2019-nCoV epidemic threat of novel coronaviruses to global health - The latest 2019 novel coronavirus outbreak in Wuhan, China. Rev. Elservier., 91 (2020) 264-266., Jan. 2020. doi: https://doi.org/10.1016/j.ijid.2020.01.009. Access in: 16 ago.2020.

6. NA, Z et al., A Novel Coronavirus from Patients with Pneumonia in China, 2019. Mag. N Engl J Med ; 382(8): 727-733., february, 2020. doi:10.1056/nejmoa2001017.

7. MINISTÉRIO DA SAÚDE (BR). Diretrizes para Diagnóstico e Tratamento da Covid19 Versão 1 Secretaria de Ciência, Tecnologia, Inovação e Insumos Estratégicos em Saúde - SCTIE. Brasília-DF abril 2020. p 398. Access en: 16 jun.2020..

8. MORENO, M, et al., Cardiovascular repercussions of infection by the new SARSCoV-2 coronavirus (COVID-19). Mag.CorSalud, [S.1.], v. 12, n. 1, p. 3-17, abr. 2020. doi:article/view/588/1113. Access en: 16 jun.2020.

9. MINISTÉRIO DA SAÚDE (BR). Protocolo de Manejo Clínico do Coronavírus (Covid-19) na Atenção Primária à Saúde: Diretrizes para Diagnóstico e Tratamento da Covid-19 Versão 9. Secretaria de Atenção Primária à Saúde (SAPS) Brasília-DF maio2020. p 41. Access en: 16 jun.2020.

10. CHATE, RC et al., Presentation of pulmonary infection on CT in COVID-19: initial experience in Brazil. J. bras. pneumol. [Internet]; 2020;46(2):e20200121. Apr., 2020. doi:https://doi.org/10.36416/1806-3756/e20200121. Access in: 16 ago.2020.

11. LANA, Raquel Martins et al., The novel coronavirus (SARS-CoV-2) emergency and the role of timely and effective national health surveillance. Cad. Saúde Pública [Internet]; 36(3):e00019620. Epub., Mar, 2020. doi: https://doi.org/10.1590/0102-311x00019620. Access in: 16 ago.2020.

12. MEHRA, MR, et al., Cardiovascular Disease, Drug Therapy, and Mortality in Covid19. Mag. N Engl J Med., 382(25): e102, Jun2020.

doi:/doi/full/10.1056/NEJMoa2007621. Access en: 16 jun.2020. 
13. WANG, D, et al. Clinical Characteristics of 138 Hospitalized Patients With 2019 Novel Coronavirus-Infected Pneumonia in Wuhan. JAMA., China. 323(11):10611069. Feb. 2020. doi:10.1001/jama.2020.1585. Access in: 16 ago. 2020.

14. WORLD, Health Organization. New coronavirus (2019-nCoV): status report- 15. Feb, 2020. Available in: https://www.who.int/docs/default-source/coronaviruse/situationreports/20200204-sitrep-15-ncov.pdf. Access in: 16 ago. 2020.

15. SUN, P, et al. Understanding of COVID-19 based on current evidence. Mag. $J$ MedVirol. 92:548-551, 2020. doi:https://doi.org/10.1002/jmv.25722. Access en: 16 jun.2020.

16. HAN, Y, et al. Society for Cardiovascular Magnetic Resonance (SCMR) guidance for the practice of cardiovascular magnetic resonance during the COVID-19 pandemic. $J$ Cardiovasc Magn Reson 22., 26 (2020)., Apr., 2020.doi:https://doi.org/10.1186/s12968-020-00628-w. Access in: 16 ago.2020.

17. HAN, Y, et al., Expert Consensus on Principles of Clinical Management of Patients With Severe Emergent Cardiovascular Diseases During the COVID-19 Epidemic. Mag. Circulation; 141(20): e810-e816, may.2020 May. doi:10.1161/CIRCULATIONAHA.120.047011. Access en: 16 jun.2020.

18. ELKIND, MSV; Harrington RA; Benjamin IJ. The Role of the American Heart Association in the Global COVID-19 Pandemic. Circulation. 2020;141(15):e743-e745. doi: 10.1161 / CIRCULATIONAHA.120.046749. Access in: 16 ago. 2020.

19. ASKIN, Lutfu; Tanriverdi Okan; Askin Husna Sengul. The Effect of Coronavirus Disease 2019 on Cardiovascular Diseases. Arq. Bras. Cardiol. [Internet]. 2020 Mai 2020. 114(5):817-822. doi: http://dx.doi.org/10.36660/abc.20200273. Access in: 16 ago. 2020 .

20. CHENG, P, et al.,Cardiovascular Risks in Patients with COVID-19: Potential Mechanisms and Areas of Uncertainty. Curr Cardiol Rep. 22, 34 (2020). doi:/10.1007/s11886-020-01293-2. Access en: 16 jun.2020.

21. FREDERIC, S, et al. Use of drugs with a potential cardiac effect in the definition of SARS-CoV-2 infection. Elsevier Masson France., França. 113 (5): 293-296, mai., 2020. doi: 10.1016/ j.acvd.2020.04.003. Access in: 16 ago. 2020. 
22. GHEBLAWI, M, et al., Enzima Conversora de Angiotensina 2: Receptor SARS-CoV-2 e Regulador do Sistema Renina-Angiotensina; Comemorando o $20^{\circ}$ aniversário da descoberta do ACE2. Circ Res., 126: 1456-1474., apr., 2020. doi:/10.1161/CIRCRESAHA.120.317015. Access en: 12 jun.2020.

23. ZHOU F, et al. Clinical course and risk factors for mortality of adult inpatients with COVID-19 in Wuhan, China: a retrospective cohort study. Lancet. 2020; 395: 105462.,march, 2020. doi:/10.1016/S0140-6736(20)30566-3. Access en: 16 jun 2020.

24. AKHMEROV, A; Marbán E. COVID-19 and the Heart. Circ Res., 126(10): 14431455, mai., 2020. doi:/10.1161/CIRCRESAHA.120.317055. Access en: 12 jun.2020.

25. ZHENG, YY, et al. COVID-19 and the cardiovascular system. Nat Rev Cardiol. China. 17, 259-260 (2020). doi:/10.1038/s41569-020-0360-5. Access in: 14 jun. 2020.

26. R, Ferrari; G. Di Pasquale; C. Rapezzi. Commentary: What is the relationship between Covid-19 and cardiovascular disease?. Commentary., 310(2020) P167-168., July 2020. doi: https://doi.org/10.1016/j.ijcard.2020.03.074. Access in: 16 ago.2020.

27. MUSHTAQ, H, et al., Structural Variations in Human ACE2 may Influence its Binding with SARS-CoV-2 Spike Protein. J Med Virol., Pakistan.apr., 2020. doi:https://doi.org/10.1002/jmv.25832_Access en: 12 jun.2020.

28. GIUSEPPE, Mancia, et al., Renin-Angiotensin-Aldosterone System Blockers and the Risk of Covid-19. N Engl J Med 2020; 382: 2431-2440., Jun, 2020. doi:10.1056/NEJMoa2006923. Access in: 16 ago.2020.

29. TOUYZ RM, Li H, Delles C. ACE2 the Janus-faced protein - from cardiovascular protection to severe acute respiratory syndrome-coronavirus and COVID-19. Clin Sci (Lond)., United Kingdom. 134 (7): 747-750apr., 2020. doi:10.1042/CS20200363. Access in: 12 jun. 2020.

30. IACCARINO, G, et al., Renin-Angiotensin System Inhibition in Cardiovascular Patients at the Time of COVID19: Much Ado for Nothing? A Statement of Activity from the Directors of the Board and the Scientific Directors of the Italian Society of Hypertension.High Blood Press Cardiovasc Prev., 27, 105-108 (2020). doi:10.1007/s40292-020-00380-3. Access en: 12 jun.2020. 